Supporting Information

\title{
Physicochemical Design Principles Enabling High-Energy and -Power Low-Cost Na Storage Materials
}

Hyungjun Kim,${ }^{\dagger}$ Myungkyu Kim,${ }^{\dagger}$ Duho Kim,${ }^{*, \downarrow}$ and Maenghyo Cho ${ }^{*, \dagger}$

†Department of Mechanical and Aerospace Engineering, Seoul National University, Gwanakro 1, Gwanak-gu, Seoul 08826, Republic of Korea

Department of Mechanical Engineering, Kyung Hee University, 1732, Deogyeong-daero, Giheung-gu, Yongin-si, Gyeonggi-do, 17104, Republic of Korea

*E-mail: duhokim@khu.ac.kr (Prof. D. Kim); mhcho@snu.ac.kr (Prof. M. Cho). 


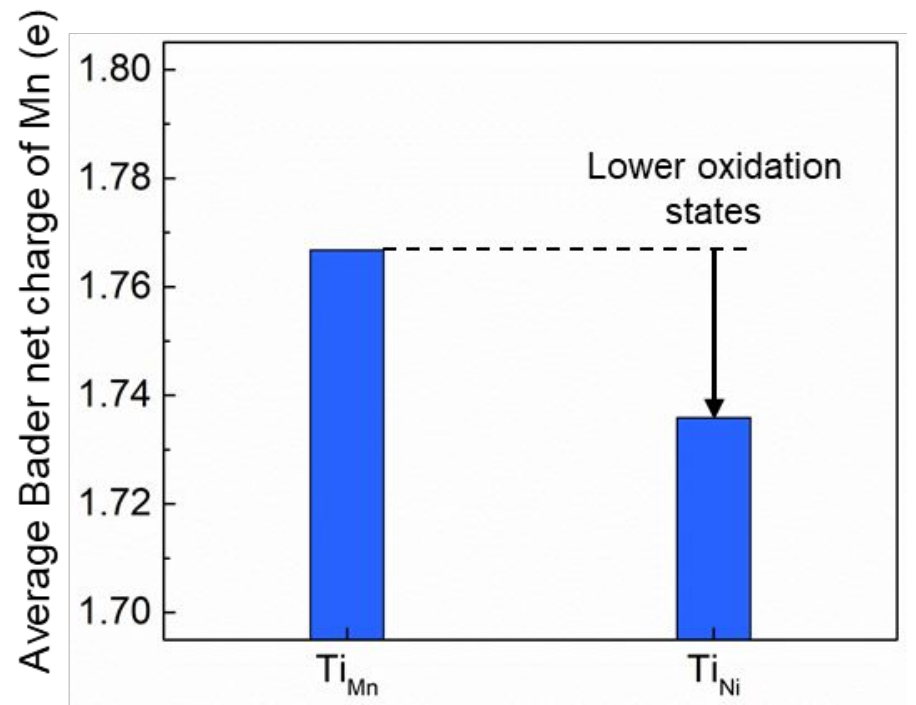

Figure S1. The average Bader net charge of $\mathrm{Mn}$ in $\mathrm{Na}\left[\mathrm{Ni}_{0.5} \mathrm{Mn}_{1.5}\right] \mathrm{O}_{4}$ of $\mathrm{Ti}$ substituting $\mathrm{Mn}$ $\left(\mathrm{Ti}_{\mathrm{Mn}}\right)$ or Ti substituting $\mathrm{Ni}\left(\mathrm{Ti}_{\mathrm{Ni}}\right)$. 


\begin{tabular}{ccccc}
\hline $\begin{array}{c}\text { Average } \\
\text { Bader net } \\
\text { charge }(e)\end{array}$ & $\mathrm{Na}_{1-\mathrm{x}}\left[\mathrm{Ni}_{0.5} \mathrm{Mn}_{1.375} \mathrm{Ti}_{0.125}\right] \mathrm{O}_{4}$ & $\mathrm{Na}_{1-\mathrm{x}}\left[\mathrm{Ni}_{0.5} \mathrm{Mn}_{1.5}\right] \mathrm{O}_{4}$ \\
\cline { 2 - 5 } $\mathrm{x}=0.0$ & $\mathrm{x}=1.0$ & $\mathrm{x}=0.0$ & $\mathrm{x}=1.0$ \\
\hline $\mathrm{Ni}$ & 1.19 & 1.36 & 1.19 & 1.37 \\
\hline $\mathrm{Mn}$ & 1.77 & 1.75 & 1.78 & 1.75 \\
\hline $\mathrm{Ti}$ & 2.28 & 2.27 & - & - \\
\hline
\end{tabular}

Table S1. Calculated average Bader net charges of $\mathrm{Ni}, \mathrm{Mn}$, and $\mathrm{Ti}$ in $\mathrm{Na}_{1}$ ${ }_{\mathrm{x}}\left[\mathrm{Ni}_{0.5} \mathrm{Mn}_{1.375} \mathrm{Ti}_{0.125}\right] \mathrm{O}_{4}$ and $\mathrm{Ni}$ and $\mathrm{Mn}$ in $\mathrm{Na}_{1-\mathrm{x}}\left[\mathrm{Ni}_{0.5} \mathrm{Mn}_{1.5}\right] \mathrm{O}_{4}$. 


\begin{tabular}{lcc}
\hline & $\mathrm{Na}\left[\mathrm{Ni}_{0.5} \mathrm{Mn}_{1.5}\right] \mathrm{O}_{4}$ & $\mathrm{Na}\left[\mathrm{Ni}_{0.5} \mathrm{Mn}_{1.375} \mathrm{Ti}_{0.125}\right] \mathrm{O}_{4}$ \\
\hline $\mathrm{NaO}_{6}$ volume $\left(\AA^{3}\right)$ & 15.485 & 15.753 \\
\hline
\end{tabular}

Table S2. The volume of $\mathrm{NaO}_{6}$ octahedron at the intermediate state, which is shown in Figure $4 \mathrm{~d}$ for $\mathrm{Na}\left[\mathrm{Ni}_{0.5} \mathrm{Mn}_{1.5}\right] \mathrm{O}_{4}$ and Figure $4 \mathrm{e}$ for $\mathrm{Na}\left[\mathrm{Ni}_{0.5} \mathrm{Mn}_{1.375} \mathrm{Ti}_{0.125}\right] \mathrm{O}_{4}$, during the sodium-ion migration. 